\title{
Pupil Dynamics in Hypoxic Conditions: Caudwell Xtreme Everest Results
}

\author{
Raj S. Dattani, ${ }^{1,2,4}$ Casey B. Swerner, ${ }^{1,3}$ Lucy R. Wyatt, ${ }^{4}$ Nikki E. Kolfschoten, ${ }^{1,5}$ Mark \\ E.G. Edsell, ${ }^{1,6}$ Christopher H.E. Imray, ${ }^{1,7}$ Michael P.W. Grocott, ${ }^{1,8,9}$ Hugh E. \\ Montgomery, ${ }^{1,10}$ and Mark H. Wilson ${ }^{1,11,12,13}$ on behalf of the Caudwell Xtreme Everest \\ Research Group
}

${ }^{1}$ Centre for Altitude, Space \& Environment Medicine (CASE Medicine), Portex Unit, Institute of Child Health, University College London, London, United Kingdom.

${ }^{2}$ St Peter's College, University of Oxford, Oxford, United Kingdom.

${ }^{3}$ St John's College, University of Cambridge, Cambridge, United Kingdom.

${ }^{4}$ School of Biological and Chemical Sciences, Queen Mary University of London, London, United Kingdom.

${ }^{5}$ Department of Surgery, Leiden University Medical Centre, Leiden, The Netherlands.

${ }^{6}$ St George's Hospital, London, United Kingdom.

${ }^{7}$ Department of General \& Vascular Surgery, University Hospitals Coventry \& Warwickshire Trust, Coventry, United Kingdom.

${ }^{8}$ Integrative Physiology and Critical Illness Group, Division of Clinical and Experimental Science, Faculty of Medicine, University of Southampton, Southampton, United Kingdom.

${ }^{9}$ Anaesthesia and Critical Care Research Unit, University Hospital Southampton NHS Foundation Trust, Southampton, United Kingdom.

${ }^{10}$ Institute of Human Health and Performance, $4^{\text {th }}$ Floor, Rockefeler Building, 21 University St, London WC1E 6DE, London, United Kingdom.

${ }^{11}$ The Traumatic Brain Injury Centre, Department of Neurosurgery, St Mary's Hospital, Imperial College Healthcare NHS Trust, London, United Kingdom.

${ }^{12}$ Faculty of Medicine, Imperial College London, London, United Kingdom.

${ }^{13}$ London's Air Ambulance, The Helipad, The Royal London Hospital, Barts Health NHS Trust, London, United Kingdom.

Address correspondence to:

Raj S. Dattani

Centre for Altitude, Space \& Environment Medicine (CASE Medicine)

Portex Unit

Institute of Child Health

University College London

UK

E-mail: rajdattani@gmail.com

Received February 7,2014

accepted in final form May 13, 2014

PUPIL OCULOMOTOR DYNAMICS ARE USED as an indirect measure of neurological function. We previously reported a reduction in pupil aperture change (PAC) and constriction velocity (CV) within 1 hour of arrival at high altitude (3450 m and $4770 \mathrm{~m}$ ), with these changes reverting to normal over subsequent days (Wilson et al., 2008). We here extend these observations as part of the Caudwell Xtreme Everest expedition (ascent profile and methodology as previously described (Levett et al., 2010).

Pupil dynamics were measured in both eyes using a handheld ForSite digital pupillometer, (Neuroptics, Irvine, USA) at 75m (London), within 2-3 days post arrival, during the morning, at 3500 m (Namche Bazaar) and 5300 m (Everest Base Camp). Of 222 possible subjects, confounding medical conditions or missing measurements left 174 subjects' data being eligible for analysis. No subjects took medication for high altitude illness at the time of, or prior to, 
pupillometry. Data from one was excluded, being an outlier (with values falling over 6 standard deviations below the mean for the rest of the sample) at all three altitudes. Pupil responses to altitude were symmetrical; mean ( \pm standard deviation) difference between left and right eye in PAC was $0.1 \pm 5.16 \%(t=-0.43, d f=461, p=0.666)$, and CV $0.02 \pm 0.4175 \%(t=-1.15$, $\mathrm{df}=460, p=0.2499)$. Given this symmetry, the average change/velocity were used as the dependent variables for purposes of data analysis. The relationships between peripheral arterial oxygen saturation $\left(\mathrm{SaO}_{2}\right)$ and both PAC and $\mathrm{CV}$ were examined through linear mixed effects models, including inter-individual variability as a random effect. Analysis was carried out using Ime4 package (Bates et al. 2013) of R for Mac version 3.0.2 (R Core Team, 2013). Models were fitted by maximum likelihood with and without oxygen saturation as a fixed effect and then compared with likelihood-ratio tests to obtain $p$-values; the final adequate models were then refitted using Restricted Maximum Likelihood.

$\mathrm{SaO}_{2}$ fell with ascent, and with it PAC (averaged across both eyes) (chi-squared=24.09, d.f. $=4, p<0.0001$ ) and CV (chi-squared=26.67, d.f. $=4, p<0.0001$ ), albeit with a small effect size (Pearson's $r=0.12$ and $0.15, p=0.009$ and 0.010 respectively; Fig. $1 \mathrm{a}$ and $1 \mathrm{~b}$ respectively).

These findings are consistent with those we previously reported, the modest effect size perhaps relating to the slower arrival at $3450 / 3500 \mathrm{~m}$ (in the past study, arrival was by air), and the 2-3 days of acclimatization allowed in comparison to the immediate measurements made in our past study. While the association of hypoxia with these pupil dynamics may be due to a direct hypoxic effect, other causal factors cannot be excluded. For instance, rising intracranial pressure (for which retinal venous congestion might prove a helpful index c.f (Wilson et al., 2013)) can cause compression of parasympathetic oculomotor nerve fibers. The symmetrical nature of our results across both eyes may support this hypothesis. Notably, our findings contrast with Schultheiss and colleagues (Schultheiss et al., 2014), who found that both CV and PAC increased as $\mathrm{SaO}_{2}$ decreased. Although Schultheiss and colleagues argue that differences in methodology and pupillometer model are important variables, our results are uniquely demonstrate consistent effects over successive trials with a consistent methodology. Furthermore, the results we now report are based on a cohort significantly larger than studied before (Cymerman et al. (2005), $n=18$; Wilson et al. (2008), $n=17$; Schultheiss et al. (2014), $n=14)$. Given these differences, further studies relating to the time-course and cause of pupillary changes at altitude are advocated. Given the use of pupil dynamics as a measure of neurological function as a part of the Glasgow Coma Scale, further work is of particular clinical relevance.

\section{References}

D Bates, M Maechler and B Bolker (2013). Ime4: Linear mixed-effects models using S4 classes. R package version 0.999999-0. URL: http://CRAN.R-project.org/package=Ime4 A Cymerman, SR Muza, AL Friedlander, CS Fulco and PB Rock. (2005). Saccadic velocity and pupillary reflexes during acclimatization to altitude $4300 \mathrm{~m}$. Aviation Space Environ Med. 76:627-634.

DZH Levett, DS Martin, MH Wilson, K Mitchell, S Dhillon, F Rigat, HE Montgomery, MG Mythen and MPW Grocott. (2010). Design and conduct of Caudwell Xtreme Everest: An observational cohort study of variation in human adaptation to progressive environmental hypoxia. BMC Medical Res Methodol. 10:98-98.

R Core Team. (2013). R: A language and environment for statistical computing. $R$ Foundation for Statistical Computing, Vienna, Austria. ISBN 3-900051-07-0,

URL: http://www.Rproject.org/.

M Schultheiss, K Schommer, A Schatz, B Wilhelm, T Peters, MD Fischer, E Zrenner, KU BartzSchmidt, F Gekeler and G Willmann. (2014). Pupillary light reaction during high altitude exposure. K Stieger. ed. PloS ONE 9:e87889.

MH Wilson, I Davagnanam, G Holland, RS Dattani, A Tamm, SP Hirani, N Kolfschoten, L Strycharczuk, C Green, JS Thornton, A Wright, M Edsell, ND Kitchen, DJ Sharp, TE Ham, A Murray, CJ Holloway, K Clarke, MPW Grocott, HE Montgomery, Imray CHE for the Birmingham 
Medical Research Expeditionary Society and Caudwell Xtreme Everest Research Group. (2013). Cerebral venous system and anatomical predisposition to high-altitude headache. Ann Neurol. 73:381--389.

MH Wilson, M Edsell, C Imray, Wright A and the Birmingham Medical Research Expeditionary Society. (2008). Changes in pupil dynamics at high altitude-An observational study using a handheld pupillometer. High Alt Med Biol. 9:319-325. 